\title{
LCMs and the liver: additional surveillance
}

A distinct population of macrophages in the liver has been identified and characterized. These liver capsular macrophages (LCMs) are different phenotypically from Kupffer cells (the predominant liver-resident macrophages) and aid immunosurveillance by sensing and restricting intrahepatic bacterial dissemination.

The liver is a key immunological organ with a high density of immune cells and is exposed to circulating antigens and endotoxins regularly. In this new study, the researchers used two-photon microscopy in mouse models to capture intravital images of cellular interactions within liver tissue to investigate the function and role of LCMs.

A distinct subset of liver-resident macrophages, the LCMs, were identified in the hepatic capsule under a layer of mesothelial cells. Morphologically, these LCMs formed a cellular network at the interface between the liver parenchyma and the peritoneal cavity and had long dendrites that scanned the environment and extended along and around collagen fibres. Furthermore, transcriptomic analysis revealed that the gene expression pattern of LCMs was more closely related to that of dermal or gut macrophages than to that of Kupffer cells.

As a result of experiments in mice, the investigators determined that LCMs were bone-marrow derived and could be replenished from circulating monocytes. Hepatic accumulation of these cells accelerated during the postnatal period at weaning, which coincided with the establishment of the gut microbiota (LCM densities were lower in germ-free mice than in specific-pathogen-free mice).

Importantly, LCMs sensed bacteria and controlled the influx of intraperitoneal bacteria into the liver via the recruitment of neutrophils. When fluorophoreexpressing bacteria (BCG-mCherry) were injected into mice intravenously they were

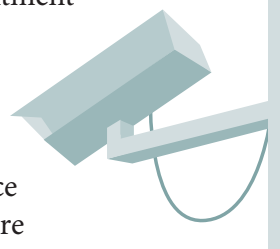
mostly associated with Kupffer cells, but when injected intraperitoneally, some of the BCG-mCherry bacteria were associated with LCMs. Moreover, when BCG-mCherry bacteria were applied directly to the capsule, imaging revealed LCM dendrites extended towards the bacteria, suggesting that the bacteria were sensed. Finally, neutrophils were recruited to the capsule after intraperitoneal injection of BCGmCherry and remained close to LCMs. Specific depletion of LCMs was associated with reduced neutrophil recruitment to the liver capsule and increased hepatic bacterial counts.

"Our finding of this novel cell type [LCMs] was really exciting as it meant that Kupffer cells were not the only macrophage population residing in the liver," note authors

David Bowen and Patrick Bertolino. The researchers now plan to explore how LCMs contribute to the attenuation or promotion of immune responses in the liver, as well as investigate whether this unique macrophage population has other functions in liver injury and disease.

Katrina Ray

ORIGINAL ARTICLE Sierro, F. et al. A liver capsula network of monocyte-derived macrophages restricts hepatic dissemination of intraperitoneal bacteria by neutrophil recruitment. Immunity 47 , 374-388 (2017)

FURTHER READING Heymann, F. \& Tacke, F. Immunology in the liver — from homeostasis to disease. Nat. Rev. Gastroenterol. Hepatol. 13, 88-110 (2016) 